\title{
La Filosofía Política de Alejandro Serrano Caldera ${ }^{1}$
}

Recibido: 13.09.2017 / Aprobado: 25.09.2017

Por Erwin Silva

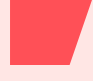

\section{Resumen}

El propósito de este trabajo consiste en analizar la red conceptual que opera en el discurso filosófico político del pensador nicaragüense Alejandro Serrano Caldera. Además mostrar las propuestas y definiciones que se encuentran en algunas de sus obras que compendian los temas de su filosofía política para Nicaragua y el aporte a la filosofía latinoamericana desde la vertiente de una filosofía de la conciencia y de la libertad.

Palabras clave: Filosofía política, nación, Estado de derecho, pensamiento latinoamericano.

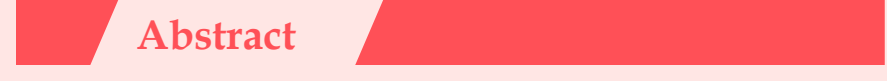

The purpose of this paper is to analyze the conceptual network that operates in the political philosophical discourse of the Nicaraguan thinker Alejandro Serrano Caldera. In addition to show the proposals and definitions found in some of his works that summarize the themes of his political philosophy for Nicaragua and the contribution to Latin American philosophy from the perspective of a philosophy of consciousness and freedom.

Key words: Political philosophy, nation, rule of law, Latin American thought.

Alejandro Serrano Caldera (Nicaragua, 1938) es un filósofo latinoamericano del siglo XX cuyo pensamiento ha sido adscrito a lo que se ha denominado Filosofía de la Liberación. ${ }^{3}$ Aunque como pensador político puede ser caracterizado como realista y moderno, éstos son rasgos que ha trazado con su labor pensante partiendo de su propia realidad histórica y basada en algunas de las categorías y teorías políticas de la Modernidad europea, particularmente Ch. de Montesquieu,

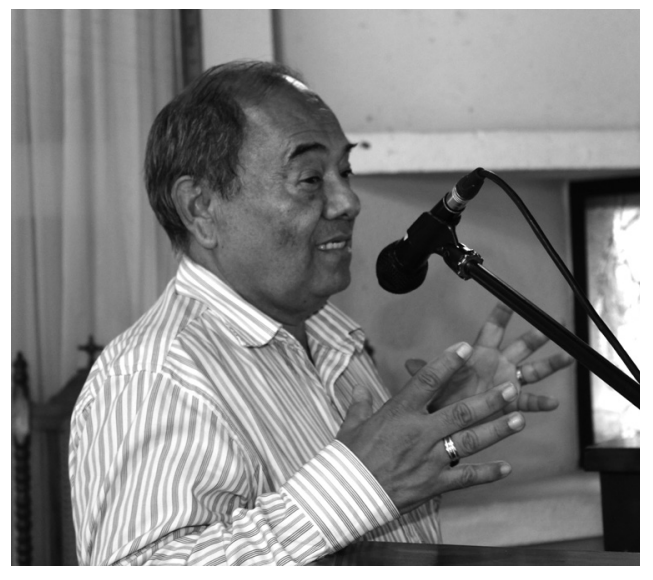

Maestro Erwin Silva.
El pensador Miguel Rojas Gómez afirma que el corpus de las ideas filosóficas de Serrano Caldera puede calificarse como una nueva filosofía de la conciencia y de la libertad. ${ }^{4}$

También debe decirse que hay un momento del derrotero de la obra de Serrano Caldera que muestra desde su punto de partida una reflexión propia generada en el nudo epistemológico Hegel-Marx. De igual manera es posible rastrear en su pensamiento la huella de Federico Nietzsche, a quien califica como el más radical de los filósofos modernos.

Conferencia magistral dictada en el marco de la VI Cátedra "Alejandro Serrano Caldera", organizada por la Escuela de Ciencias Jurídicas y Políticas de la UPOLI. Managua, 27 de septiembre de 2016.

2 Docente Investigador. Coordinador del Área de Educación para la Paz y los Derechos Humanos en el Instituto de Investigaciones y Acción Social "Martin Luther King" de la Universidad Politécnica de Nicaragua (UPOLI). email: erwinjotasil@gmail.com

3 Sánchez Rubio, David. Filosofía, Derecho y Liberación en América Latina.Desclée de Brouwer, Bilbao, 1999.

4 Rojas Gómez, Miguel en: Una nueva filosofía de la conciencia y la libertad. Editorial Universitaria, Managua, Nicaragua, 1993. 


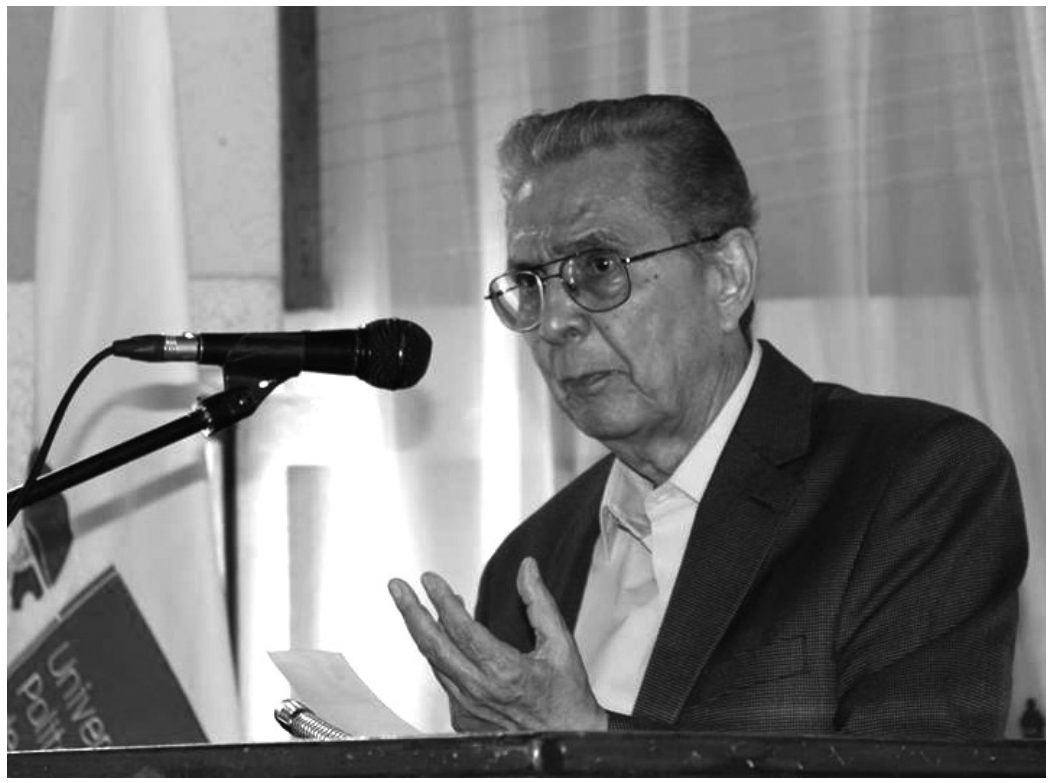

Dr. Alejandro Serrano Caldera, ofreciendo sus palabras en la conferencia magistral.

Así que la trilogía Hegel, Marx y Nietzsche es muy productiva en el opus de nuestro filósofo, trascendente en todos los términos para nuestra historia de las ideas filosóficas y políticas.

En el presente texto no se quiere efectuar más que la tarea de mostrar y analizar una red conceptual en el marco del discurso filosófico de Alejandro Serrano Caldera. Discurso es lo que se dice. Utilizo la noción de discurso de Michel Foucault, en este caso, porque con este concepto se superan problemas tales como la existencia de una filosofía latinoamericana o de la identidad. ${ }^{5} \mathrm{La}$ posibilidad que se abre con la aplicación del constructo del discurso foucaultiano, nos deja ver que la filosofía política de Serrano Caldera de hecho es un pensamiento producido y progresivamente sistematizado, concibiendo para Nicaragua y para América Latina un proyecto sin olvidar los reinos de Utopía, que bien vale repetir que América tanto como Nicaragua, oscilan entre el proyecto y la utopía.

Con el trabajo de pensamiento y la obra es posible, como he dicho ya, tejer una red de conceptos, que no sólo devienen con nuevas cargas significativas, sino que son inéditos en nuestro ámbito político y filosófico, lo que constituye un aporte para nuestra cultura política y para el ejercicio de una nueva ética política.

\section{El concepto de dialéctica}

Al efectuar el estudio del pensamiento de Serrano Caldera, debemos comenzar en su genealogía por el análisis del concepto de dialéctica que en él es una herramienta que aplica como crítica social, política y filosófica.

Del concepto dialéctica sabemos ha evolucionado desde Sócrates, Platón y Aristóteles y que en el primero es el arte de la pregunta con la que se extrae una verdad en el diálogo con el amigo o el oponente, en el segundo es el instrumento de acceso al mundo noético de las puras eídoses y en el tercero, una lógica configurada para ejercer la demostración deductiva de una verdad. En G. W. F. Hegel, en tanto es una síntesis de los opuestos, la Idea en sí y para sí, el Espíritu que se aliena en Naturaleza y se reconcilia consigo mismo. La dialéctica en Hegel es una ontología. Para Karl Marx, en cambio, la dialéctica no es de la idea ni del espíritu sino de la realidad, una auto dinamia de la materia. 
...Viene de la página 24

Alejandro Serrano Caldera es precisamente un filósofo que ha contribuido al estudio del pensamiento moderno y como tal, nos lega Introducción al pensamiento dialéctico (1976) donde afirma que: En la formación de la ciencia moderna y del pensamiento dialéctico concurren la razón cartesiana, el humanismo naturalista de Rousseau, la dialéctica idealista de Hegel y el materialismo dialéctico de Marx. ${ }^{6}$

Y es en este estudio de la génesis del pensamiento filosófico y la realidad histórica vistos como procesos, particularmente de

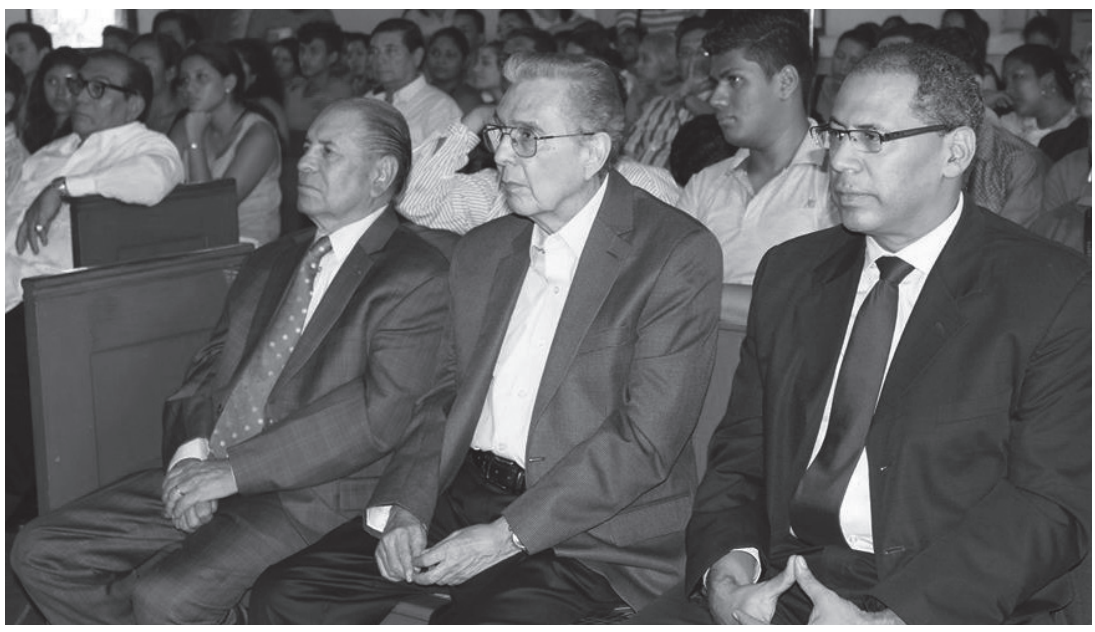
Latinoamérica, donde se ve la aplicación creadora y no mecanicista de la dialéctica que Alejandro Serrano Caldera logra gestar las bases de un discurso, de un pensamiento propio y una visión política para la nación nicaragüense.

\section{Dialéctica y Enajenación}

Alejandro Serrano Caldera, al desplegar el concepto de enajenación con el de dialéctica, realiza, a mi ver, una doble operación discursiva: primero, vincula dos conceptos claves de la filosofía hegeliana y sobre todo marxista que sirven para explicar la situación del trabajador moderno como productor de mercancías de las que no es dueño, propiciada por una división del trabajo y una estructura de clases sociales donde la burguesía es la propietaria de los medios de producción, cuestión que K. Marx deja establecida desde 1844 en sus juveniles y célebres Manuscritos Económicos y Filosóficos. Aunque la categoría enajenación o alienación cobra varios aspectos en la realidad, aspectos económicos, políticos, morales, ontológicos e incluso estéticos, como se encarga de comprobar István Meszáros en su obra clásica Marx's Theory of Alienation; ${ }^{7} \mathrm{y}$ en segundo lugar aplicarla a nuestra realidad histórica y la cultura del presente.

De ahí que los estudios de Dialéctica y filosófico. Dialéctica y enajenación como conceptos son consecuentes ya que derivan de lo más revolucionario del método de Hegel y de Marx y le sirven a nuestro pensador como "instrumentos interpretativos de la cultura, la ciencia, la filosofía y la teoría del conocimiento moderna y contemporánea". ${ }^{8}$

En estos estudios se destacan también sus tesis sobre filosofía en las que señala las limitaciones del pensamiento latinoamericano. Serrano Caldera dice que tenemos logros en las actividades artísticas y en algunas esferas científicas pero no en el campo de la Filosofía. Para Serrano Caldera no hay aportes a la ontología, la gnoseología ni la axiología.

La Filosofía sólo surgirá "cuando se superen los estadios específicos de nuestro desarrollo cultural, lo cual conlleva a la vez a la superación de nuestra situación histórica estructural y el desarrollo de un pensamiento crítico". Esta es una tesis similar a la sostenida por Augusto Salazar Bondy en su texto ¿Existe un filosofía de nuestra América?. ${ }^{9}$ Sin embargo, la senda que abre el pensamiento de Serrano Caldera es una prueba de esas superaciones que se piden para la existencia de una filosofía latinoamericana auténtica.

Enajenación (1979) de Alejandro Serrano Caldera, constituyen un paso en la formulación de su discurso

Serrano Caldera.Alejandro. Introducción al pensamiento dialéctico. Fondo de Cultura Económica, México, 1976.

Metzáros, Istvan. Marx' Theory of Alienation. Harper Torchbook, USA, 1972.

Serrano Caldera, Alejandro. Dialéctica y Enajenación. EDUCA, San José, Costa Rica, 1979.

Salazar Bondy, Augusto. ¿Existe una filosofía de nuestra América? Siglo Veintiuno editores. México DF, 1988. 


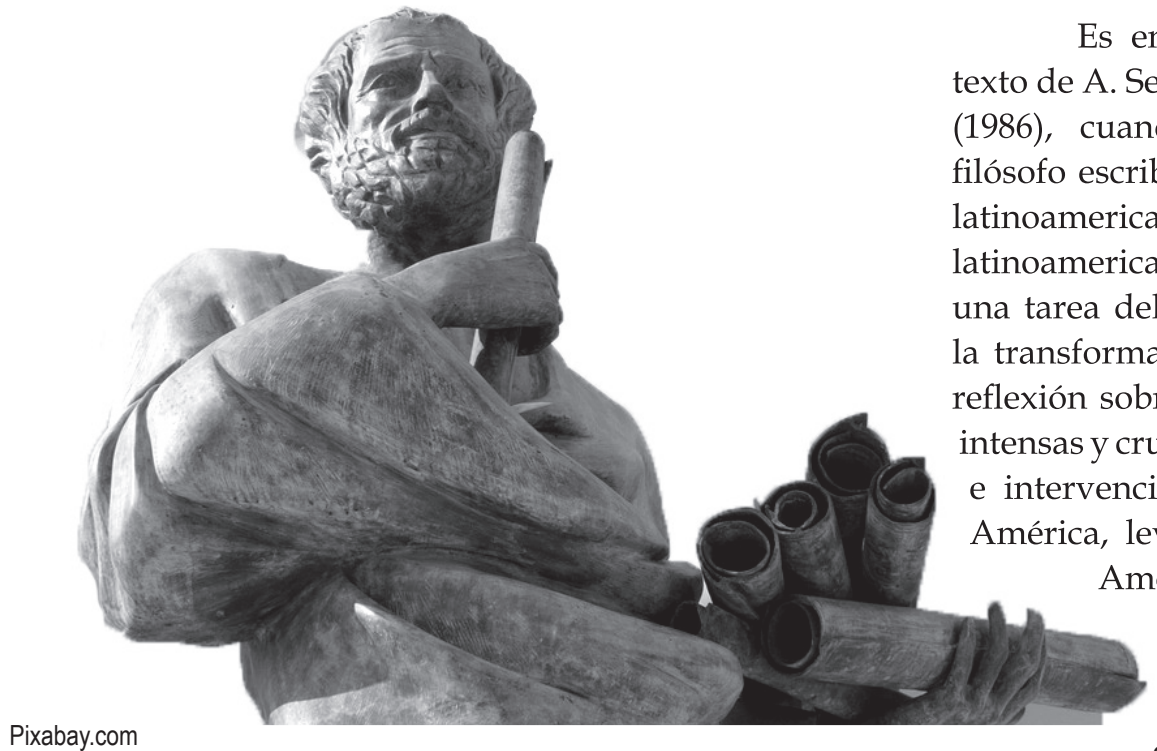

Pixabay.com

\section{Nación e Imperio}

Otra unidad del discurso filosófico de A. Serrano Caldera es la contradicción Nación e Imperio, que según entiendo, proviene de la Filosofía de la Liberación, por un lado, y del otro, de la experiencia histórica vivida, analizada e investigada por nuestro pensador. Pues en Nicaragua, ha existido un pueblo, una nación que se ha mostrado como tal en los momentos en que ha enfrentado las intervenciones militares y políticas del imperio norteamericano. Períodos claves de nuestra historia como la Guerra Nacional de 1855-56; el del liberalismo reformista de José Santos Zelaya, 1893-1909; la Guerra Campesina antiimperialista del General Augusto C. Sandino, 19261934; la Revolución Sandinista, 1979-1990, han sido tiempos de lucha e imaginación. Esto es lo que Serrano Caldera denomina una nación intermitente. ${ }^{10}$

A propósito de Nación e Imperio, Mario Carlos Casalla en Cultura Popular y Filosofía de la Liberación ${ }^{11}$ sostiene que hay una dicotomía dialéctica básica: dependencia opresora/liberación integral y es desde ahí donde se piensa el proyecto de pueblo. Así que pueblo deviene en nación por la memoria, el destino y el conflicto. La nación es voluntad de ser y el imperio voluntad de poder.
Es en esta antinomia que también aparece el exto de A. Serrano Caldera, Entre la Nación y el Imperio (1986), cuando en plena Revolución Sandinista, el filósofo escribe unos prolegómenos a una teoría del ser latinoamericano en los que se asevera que la identidad latinoamericana es una síntesis necesaria y la liberación una tarea del pueblo y éste el sujeto por excelencia de la transformación. Este libro es, en cierto modo, una reflexión sobre la historia de Nicaragua que ha sido de intensas y cruentas luchas contra las sucesivas agresiones estados Unidos de América desde una Revolución. La Historia de Nicaragua es como sostiene Serrano Caldera, "una reafirmación en la libertad", su sentido es la Libertad. La Filosofía de Serrano Caldera es más bien una Filosofía de la Libertad que se plantea unas búsquedas conducentes a la superación de la dependencia, la dominación y la hegemonía del imperio. En fin, dos términos balancean esta obra y otras anteriores, identidad y liberación, cuestiones presentes ya en sus obras como lo es Filosofía y Crisis.

Ciertamente las posiciones del filósofo A. Serrano Caldera han cambiado así como sus conceptos y la conjugación de las categorías que han pasado del trinomio dependencia / liberación / revolución, ésta última como solución y alternativa a la opresión, el subdesarrollo y la desintegración nacional a una filosofía del desarrollo y una ética de los valores que nos hacen pensar en la condición humana, lo que da lugar a reflexionar en un humanismo ético o de los valores.

Aunque hoy se descree de la organicidad y la militancia de los intelectuales a lo Gramsci, debe decirse que A. Serrano Caldera por sus aportes al pensamiento latinoamericano, su análisis y su prospectiva se hace cargo de la realidad que es fuente - Latinoamérica y Nicaragua en especial, ocupan su filosofía política que en gran medida se centra en la proyección de una nación como síntesis más allá de la simple reconciliación de los opuestos, como una fundación de una realidad que surge de la unidad en la diversidad.

10 Serrano Caldera, Alejandro. Entre la Nación y el Imperio. Editorial Vanguardia, Managua, Nicaragua, 1985.

11 Casalla, Mario. En: Cultura Popular y Filosofía de la Liberación. Buenos Aires. Fernando García Cambeiro,1975. 


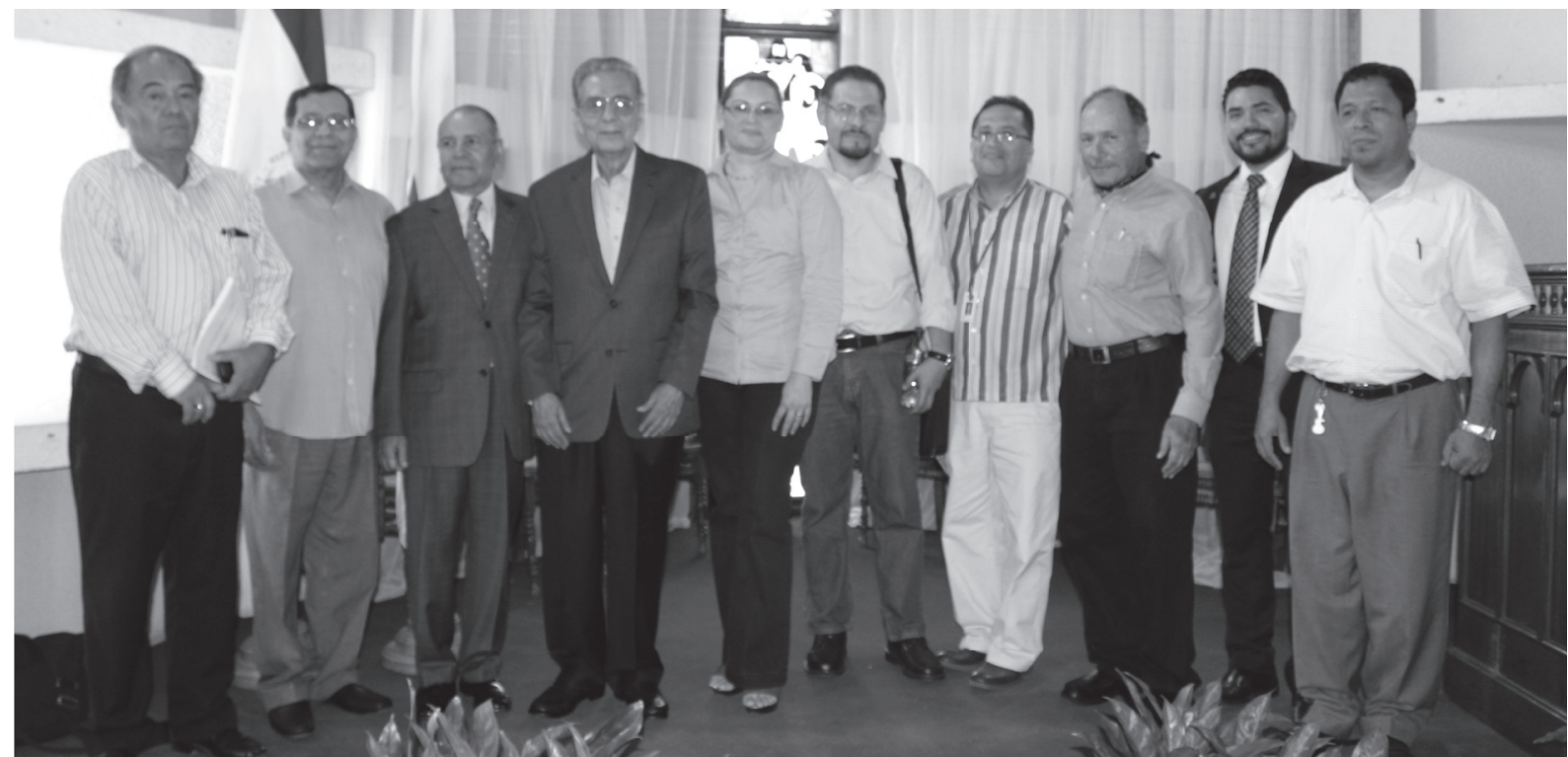

EI Dr. Alejandro Serrano Caldera con representantes académicos de la UPOLI y otras universidades. Foto: Relaciones Públicas UPOLI.

\section{La unidad en la diversidad}

Esta tesis de la Unidad en la Diversidad ${ }^{12}$ que da título a una de sus obras, no es como nos quiere hacer creer Fernanda Beigel ${ }^{13}$ que es una consigna sino una tesis con una antigua y prolongada tradición en el pensamiento filosófico occidental que va de Heráclito, Platón, y Plotino a Hegel y Marx y que en A. Serrano Caldera sirve de fundamento para pensar en una Nicaragua posible.

La unidad en la diversidad en nuestro caso representaría la construcción de una hegemonía latinoamericana que pasa por la superación de la contradicción sin síntesis que es nuestra cultura todavía. Aunque esa síntesis o múltiple determinación ya se inició para nosotros con el pensamiento filosófico latinoamericano que irrumpe con la liberación de los pueblos americanos del imperio español y de la colonización portuguesa. El pensamiento filosófico sobre la identidad, la cultura, la integración y la liberación; son muestras de esa síntesis.

Aun transido de hegelianismo y de un marxismo leído desde Hegel, A. Serrano Caldera desarrolla la tesis de la unidad en la diversidad, muy productiva para él, puesto que le sirve de plataforma para darle los fundamentos filosóficos al proyecto de nación, armonizado en el conjunto centroamericano y el concierto de América Latina, pasando por la articulación de un nuevo contrato social que supone a la vez el consenso y la democracia.

\section{La Nación: comunidad de valores}

Nación es un concepto que en el discurso filosófico de Serrano tiene, digamos, una enorme importancia porque está ligado directamente a su realidad que es Nicaragua, un país con un largo historial de violencia, "fragmentación social y desintegración de objetivos comunes". La Nación, para Alejandro Serrano Caldera, es una comunidad de valores y una tarea estratégica que cumplir para establecerla como idea capaz de movilizar hacia un ideal común.

La tarea de construir una nación se haría en relación con tres ejes principales:

Un común denominador: la cultura; un objetivo: la construcción del nuevo Estado- Nación; y una idea motriz: la participación como nación en los desafíos del mundo contemporáneo y del futuro inmediato.

12 Serrano Caldera, Alejandro. La unidad en la diversidad. Hacia una cultura del consenso, Managua, 1998.

13 Beigel, Fernanda. Derribando muros y creando realidades. IDEHU, UPOLI, CIEETS, Managua, 1999. 
Este concepto de nación también convoca, dice el propio filósofo, a una idea de la nación centroamericana como si fuese:

una comunidad de raíz y destino, de pasado y futuro, de realidad y proyecto.

Idea que también en esencia refiere a la cultura de esta región que tiene una historia comun

La Nación, forma vital y dinámica, conciencia, comunidad, ideal, proyecto, creación, esfuerzo y condensación cultural tiene una centralidad y una influencia vivificante para nuestro pensamiento filosófico-político nicaragüense, de tal manera que ante la disociación, la divergencia, la intermitencia porque Nicaragua ha sido una nación con esa característicaestá el "plano de coincidencias mínimas"14 que A. Serrano Caldera piensa que puede ser la Nación. En este punto se da lo que dice Fernando Mires en su libro "Civilidad, Teoría política postmoderna"15 que en nuestros días se da un retorno de la nación en la medida en que estamos más globalizados.

\section{El diálogo, la Nicaragua posible y el proyecto de Nación}

Al respecto el propio filósofo A. Serrano Caldera se encarga de decirnos qué obras de su autoría compendian hasta cierto punto la unidad temática de su filosofía política y estas son: La Unidad en la Diversidad, Los Dilemas de la democracia y Hacia un proyecto de Nación. ${ }^{16}$

Por esto es muy importante en primer lugar, analizar en el pensamiento filosófico político de A. Serrano Caldera, el valor del dialogo, que está fundado en la cultura, cuyo ejemplo es aquel Dialogo de Huexontzinco.

El dialogo puede ser sobre mucho temas vitales como la guerra, la paz o el estado, pero también puede empleado para conseguir el consenso y es una opción civilizada, es digamos una instrumento de la paz.

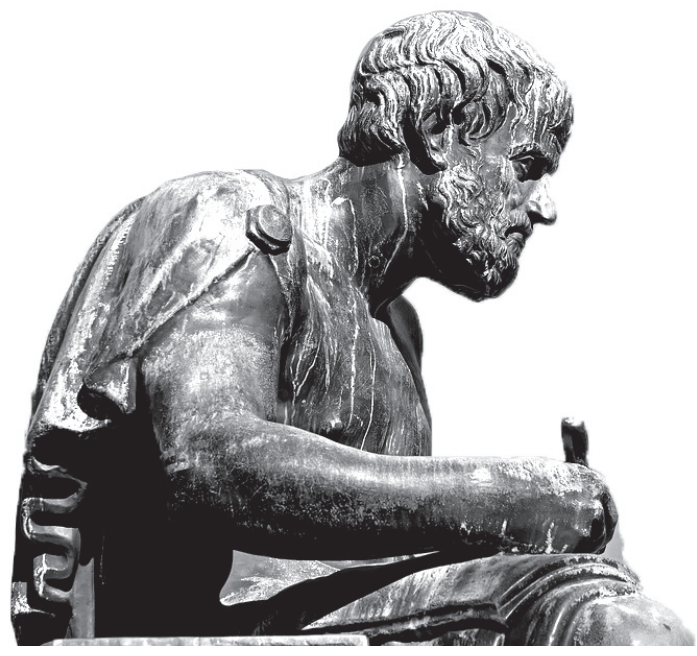

Pixabay.com

Por otra parte el filósofo Serrano Caldera ha propuesto a nosotros los nicaragüenses una Nicaragua posible:

La Nicaragua Posible - dice el filósofo- no es la Nicaragua ideal de nuestros sueños ideológicos o de nuestras utopías políticas, es la Nicaragua que todos y cada uno de nosotros podemos construir cediendo un poco de lo que constituye nuestro desiderátum político o el paradigma de nuestro modelo integral de sociedad.

Es la Nicaragua del consenso y de la democracia, la que surge de la unidad de nuestras diferencias. No es la Nicaragua homogénea. ni tampoco es la Nicaragua caótica y confrontativa, la del maniqueísmo que niega todo lo que no reproduce la propia imagen y deseos, sino la Nicaragua plural y múltiple, en la que todas la expresiones políticas tienen un espacio legítimo.

Esta Nicaragua posible es la Nicaragua fruto de la concertación y la concertación es una forma de conducta política, un estilo de conducir la política y lo político con miras a la construcción de la sociedad del futuro; pero es también un instrumento preciso para dar respuesta perentoria a los problemas apremiantes que gravitan con dramatismo sobre nuestro pueblo.

Serrano Caldera, Alejandro. La unidad en la diversidad. Hacia una cultura del consenso.Managua,1998

Mires, Fernando. Civilidad y teoría política postmoderna.

Serrano Caldera, Alejandro. Hacia un proyecto de nación. Una década de pensamiento político. Fondo Editorial CIRA, Managua, 2001. pp.7-8. 


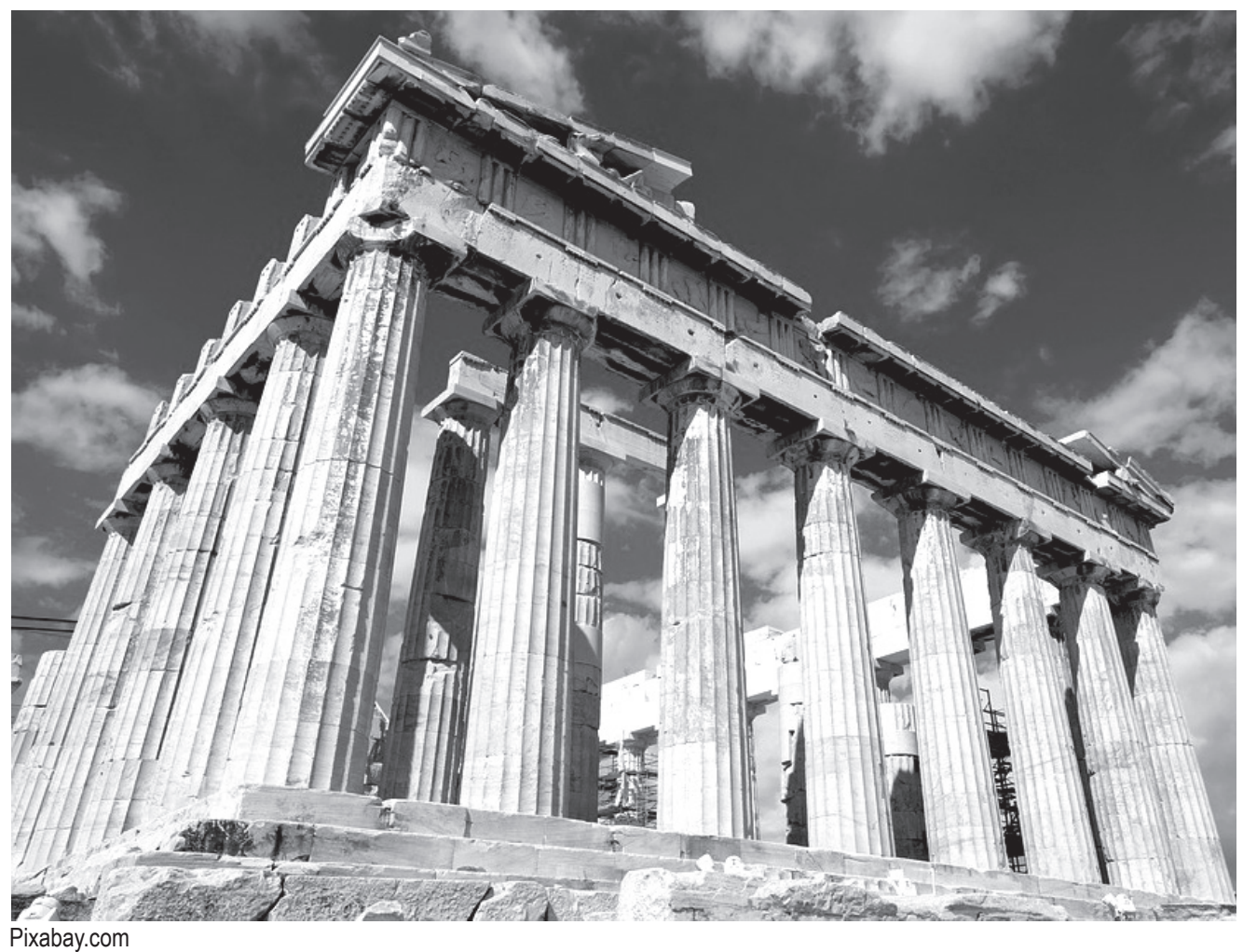

\section{La democracia, dilemas y nuevos elementos}

El $\begin{array}{r}\text { concepto } \\ \text { democracia en Serrano }\end{array}$
Caldera, articulado de tal
manera en su formación
discursiva, que resulta de
un valor determinante para
su propio discurso filosófico
y para la realidad política
efectiva en Nicaragua
ayuna de tal forma política
y agobiada por dictaduras
militares y autoritarismos
de toda laya. La democracia
para A. Serrano Caldera es un
sistema de valores, el plexo de
estos valores está constituido
por la libertad, la tolerancia,
legalidad, legitimidad y el
respeto a la diferencia.

La concertación significa un salto cualitativo sobre lo que han sido las dos expresiones dominantes de la política criolla: la confrontación o la claudicación. ${ }^{17}$

Una definición clara del proyecto nacional propuesto por nuestro filósofo que no se alcanzaría sin consenso sin identidad e integración frente la a la globalización es la que dice que es:

Un proyecto nacional es la plataforma política, económica, social y cultural, ampliamente aceptada por una sociedad determinada.

La filosofía mínima de este proyecto nacional es el consenso, el desarrollo y los valores sociales cuyo centro de todo es la dignidad de la persona humana y por supuesto la democracia.
El Estado de Derecho es a la vez causa y efecto, condición y consecuencia de la democracia. Por ello, sin Estado de Derecho no hay democracia, pues la subordinación del poder a la ley, que es la garantía de todos, desaparecería, y con ella, desaparecerán también el principio de legalidad y la regulación institucional, que origina y define el poder, que determina su intención y dirección, para dejarlo en manos de la fuerza, la ambición y el juego de los intereses personales o de grupo.

La democracia, además de un ejercicio legal e institucional, es iniciativa ciudadana.

En cierto sentido la democracia es un arte: el arte de los equilibrios (...)

La democracia no es tanto una realidad consumada como un proyecto inacabado, una búsqueda, una voluntad, una esperanza. ${ }^{18}$

17 UNAN- Managua. Memoria. La Nicaragua posible. Managua, Editorial UNiversitraia, 1991.pp.10-11

18 Serrano Caldera, Alejandro. La democracia, concepto y desafíos actuales. CLACSO. s-f. 
En todo caso la democracia debe asumirse como actitud moral que debe conciliar la libertad y la justicia. Y agrega el pensador Serrano Caldera en este texto que la democracia es una "forma de vida, de conducta y de actitud ante el mundo". En todo caso, la democracia es una cultura para Serrano Caldera, lo que indica que forma parte de nuestra vida y conducta cotidiana, es una conquista y una reconquista ética-política de cada día al decir de J. L. Aranguren. Realmente la cultura democrática es la que se establece a partir de unos valores y unas prácticas comunes en la familia, la sociedad, los países y las relaciones internacionales.

El autor nos brinda otros aspectos y elementos de su teoría de la democracia en el texto Los dilemas de la democracia $(1995)^{19}$ que es necesario abordar, porque son vitales en la comprensión del concepto, o sea lo que engloba tal noción desde su óptica. Entre estos, que la democracia no sólo deber ser concebida como democracia representativa que por demás está en crisis, porque se agota en el sufragio o el recambio de autoridades.

$\mathrm{Y}$ es que precisamente "el problema actual, en la democracia representativa es la hegemonía de las minorías a expensas de las mayorías que dicen representar". Se trata pues, de revitalizar la democracia, de identificar los nuevos sujetos sociales que la agencian y de una participación cada vez más plena, lo que requiere que la democracia sea también una democracia participativa.

Además, la democracia, según Serrano, se nutre del consenso, lo exige como complemento del ejercicio mismo de la democracia frente a la fragmentación, la tensión y la polarización que ha mostrado y muestra la realidad política de Nicaragua.

En cuanto a los nuevos sujetos de la democracia - aunque debe advertirse que se ha declarado hace algún tiempo la muerte del sujeto - son para Serrano Caldera:

las asociaciones profesionales, sindicales, juveniles, femeninas, ecológicas, culturales, artísticas, campesinas, estudiantiles, lo mismo que a instituciones como las universidades y municipios que actúan dentro del marco de sus respectivas autonomías...

Como se ve estos son grupos o segmentos de la sociedad civil que reivindican un derecho en particular o defienden un interés universal y cuya visión política se opone tanto a la idea de partido como de Estado, estas expresiones de la sociedad civil contribuyen al fortalecimiento de la democracia que la hace un sistema vivo y no de papel.

Es notorio que el filósofo Serrano conciba a la democracia como un proyecto ético, pero sus agudas observaciones de la realidad política nacional y mundial lo convierten en un analista que nos da una entera reflexión sobre la democracia cuando no tenemos otro sistema mejor. Un conjunto de atisbos, elementos y relaciones nuevos de la democracia es anotado por nuestro filósofo con gran acierto y realismo. Tal es la relación que se plantea entre la democracia y el Estado de Derecho, en tiempos del mercado absoluto y globalización dirigida.

\section{La democracia y el Estado de Derecho}

El análisis y la reflexión de A. Serrano Caldera sobre el juego de relaciones entre estos conceptos y categorías de la realidad política, cuya arqueología podemos rastrear hasta los inicios de la filosofía política moderna europea, parten de Montesquieu y de John Locke con la teoría de la separación de poderes y el equilibrio entre éstos, el principio del Estado de Derecho y la Democracia Modernos.

Como jurista, A. Serrano Caldera, presenta algunos elementos conceptuales del Estado de Derecho que hoy cobra una vigencia universal en el proceso político-histórico mundial, cuando los teóricos políticos actuales, proponen la democracia formal, el mercado y el propio estado de derecho como una trilogía fundamental para la sociedad en un solo mundo.

19 Alejandro Serrano Caldera. Los dilemas de la democracia.UAM, Managua. Nicaragua.1995 
Si nos atenemos a la definición dada por el autor, Estado de Derecho es la subordinación del poder a la Constitución y las leyes. Este es, digamos, su principio universal y, consustancial a éste, la jerarquía de la norma jurídica con la que se establece la supremacía de la ley de leyes que es la Constitución. Pero A. Serrano Caldera historiza el concepto de Estado de Derecho y lo hace en el contexto de la realidad de Nicaragua, partiendo de un estudio breve del sistema constitucional y de algunos de los artículos de la Constitución Política vigente, para concluir en un análisis de la teoría y la praxis constitucional del Estado de Nicaragua que ha sido ni más ni menos que la historia del poder, debido al sistema presidencial, el autoritarismo y la falta de conciencia de la institucionalidad de los propios nicaragüenses.

Los resultados que obtiene Serrano Caldera del estudio del Estado de Derecho en Nicaragua son útiles para la comprensión de la naturaleza política e institucional que caracteriza hasta el momento a la sociedad nicaragüense. Algunas de estas situaciones observadas reflejan una evidente crisis cultural. Por ejemplo, la proclamación invariable de derechos y libertades modernas se contradice con las prácticas políticas autocráticas, la contradicción entre el Estado y la Sociedad Civil, que se perciben como distantes, la inmediatez en los resultados de la política y la carencia de una visión de cambios estratégicos para la vida política de la nación. ${ }^{20}$

\section{Crítica de la postmodernidad}

Cuando se ha constatado filosóficamente la muerte del hombre y la deslegitimación de los metarrelatos emancipatorios, llega esta época marcada por el final de todo, que se denomina posmodernidad en el ámbito filosófico, prepara también el retorno de lo sagrado y de los antiguos mitos. Al estudiar varios autores de esta zona de tiempo, hallamos unas características que resumidas son:

1. Fin de la historia

2. Deslegitimación de los metarrelatos
3. Reivindicación de lo plural

4. Disolución del sujeto

5. Crítica a la razón

6. Predominio de la estética frente a la ética ${ }^{21}$

Pero la postmodernidad no sólo es el desvanecimiento de los arquetipos de la modernidad, es la deconstrucción de Jacques Derrida, el desmigajamiento de Emil Ciorán, el pensamiento débil de Gianni Vattimo y la melancolía de Lyotard. Hoy quizás el tema ha escandalizado mucho en los países del primer mundo y no es extraña una saturación del concepto en los mundos académicos oficiales donde se ha convertido ya en reiteración. Ronda nuevamente el espectro de Marx según Jacques Derrida.

Nuestro filósofo, Alejandro Serrano Caldera, que puede contarse entre los críticos de posmodernidad en Latinoamérica, dice que:

La postmodernidad no es sólo la deconstrucción de los paradigmas de la modernidad, de la concepción moderna del mundo y de la sustitución del lenguaje moderno, sino que es, aunque no lo proponga explícitamente y aunque en algunos casos no lo mencionen los teóricos, la formación de un nuevo sistema de vida cultural y social; de una nueva forma de producción, la producción transnacional, de una nueva organización capitalista, el mercantilismo corporativo y de una nueva concepción del Estado-Nación, a través de la transnacionalidad jurídico-institucional. ${ }^{22}$

Por supuesto, la crítica de Serrano capta que la post- modernidad es también crítica a la filosofía de Hegel y Marx y establece los vínculos existentes entre el proceso de globalización y la postmodernidad. Esto significa que por primera vez existe un solo mundo unido por unas redes tecnológicas y financieras que sobrepasan la categoría estado-nación y la identidad cultural de los pueblos.

Pero al percibir estos cambios en la conciencia mundial y en las prácticas políticas que derivan de la deconstrucción de los modelos políticos e ideológicos, A.

20 Serrano Caldera, Alejandro. Obras. Escritos sobre el pensamiento, la política y la cultura nicaragüense. Escritos jurídicos. Vol. IV. Managua. HISPAMER: CNU.2013.pp.446-451.

21 López Gil, Marta. Filosofía, Modernidad y Postmodernidad. Editorial Biblos , Buenos Aires,1996

22 Serrano Caldera, Alejandro. La filosofía ante el reto de nuestro tiempo, por una ética de los valores. Ed. Universitaria. Managua, 1994. 
Serrano realiza una crítica: la tesis del fin de la historia y del totalitarismo de mercado que lleva a nuevas conclusiones sobre el trasfondo filosófico y político del capitalismo transnacional. Estas deducciones de nuestro pensador son de una gran utilidad para el pensamiento latinoamericano actual, destinadas a superar el neoliberalismo que pretende ser universal o global y proponer alternativas a las sociedades disociadas y dominadas por un modelo de desarrollo que no es endógeno ni sostenible y mucho menos humano.

Recordemos algunos de los acontecimientos políticos relevantes de los años ochenta y noventa como es, por ejemplo, el derrumbamiento del paradigma revolucionario en la Unión Soviética que hizo pasar del stalinismo a la reforma de Mijaü Gorbachov que redescubre el mercado y la democracia, llevando al fin de las ilusiones en la línea de Marx y Lenin.

Así que cuando se desmorona el socialismo real, que ni fue socialismo ni fue real, reaparece en lo ideológico y lo filosófico en Occidente la tesis del fin de la historia como un lema basado en un capitalismo victorioso sobre dos enemigos: el fascismo y el comunismo. Como se sabe es Francis Fukuyama quien retorna a la fuente de Hegel, éste había desarrollado ya la tesis del fin de la historia. Para Hegel el fin de la historia llega con los giros tomados por la Revolución Francesa y con el establecimiento del sistema capitalista.

Ensu odisea el Espíritu según Hegel - plegándose y replegándose - enajenándose en naturaleza, historia, regresa a sí, lo que constituye el fin de la historia como experiencia humana. Fukuyama hace coincidir fin de la historia con "una justificación de histórica del capitalismo y del liberalismo. ${ }^{23}$

\section{La ética como fundamento de la política}

Con antecedentes posibles en la axiología de Max Scheler y frente a la razón instrumental, los desafíos y las posibilidades que germinan en la realidad histórica latinoamericana, Serrano Caldera, fundamenta una ética de los valores, "una ética de la racionalidad, del desarrollo y de la democracia". ${ }^{24}$

Las categorías de ética y política vistas como antagónicas o separadas a partir de la modernidad, tienen la posibilidad de mezclarse y hasta de fundirse en una ética política. En todo caso, plantearse de nuevo la reunión de estos conceptos nos retrotrae a la filosofía política clásica de Aristóteles, en la que prima el deber ser de la política y la búsqueda del bien común.

La ética individual y la ética social coincidían y la ética y la política tenían su intersección en el ámbito de la polis, dándole cohesión y consecuencia a la praxis política con relación a unos valores.

En la utopía de Platón llamada La República, la dikaiosyne o justicia es el vértice donde confluye la realización política y ética de los individuos y de la sociedad, es, digamos, el valor que se corresponde con la parte racional del ser humano que se levanta sobre el instinto y la pasión. Recordemos que en esta obra platónica la sicología y la política son planos de correspondencia con formas de gobierno perfectas o corruptibles.

Una ética de los valores en el caso de Serrano Caldera significa solidaridad frente a los valores individualistas y egoístas que no son los del egoísmo ilustrado sino los más vanos y crueles del mundo de la tecnocracia y las finanzas y que hoy hace circular el neoliberalismo y aunque parezca un retorno imposible, ética y política son enormes espacios donde es factible una intersección para producir o crear al menos el bienestar o el desarrollo a una escala humana.

\section{Humanismo ético}

Para Alejandro Serrano Caldera el hombre latinoamericano ha de asumir el destino de la humanidad total para ser realmente universal. El hombre de nuestra América debe superar su enajenación, la dominación que ha ejercido el Occidente sobre él; esta sería su dialéctica, de tal manera que:

23 Serrano Caldera, Alejandro. El doble rostro de la postmodernidad. CSUCA, San José, Costa Rica, 1994

24 Serrano Caldera, Alejandro.Op. Cit. 
La liberación y la identidad constituyen, en el momento actual, el propósito esencial de una parte de la humanidad. El sentido de la liberación está enlazado en la trama de la vida. Es la liberación de la de la explotación, de los fantasmas de la superstición, de la ideología opresora, de la enajenación. ${ }^{25}$

De esta forma en la acción de liberarse se replantea también "la estructura moral dela humanidad". Pero, para llegar a estos resultados que son de una época, veamos cómo concibe Serrano Caldera al hombre americano. En primer lugar, este hombre - visto desde la ontología y la axiología - es un ser no restaurado, desgarrado aun cuando se piense que el mestizaje racial lo ha integrado, la situación real y específica es la de una yuxtaposición de modo que "Así, en forma violenta y por la puerta de atrás, entra el hombre americano a la civilización occidental". En esto, Serrano Caldera, participa con Leopoldo Zea la visión del hombre americano al que la Conquista de los españoles rebajó el status antropológico al extremo que un Papa Católico, nos declaró hombres por la risa. Un origen de la filosofía latinoamericana está justamente allí en la autoconciencia de la enajenación, la dominación y la explotación que sería el primer paso en la dialéctica de la liberación.

Desde la ontología, el hombre americano puede concebirse como ser dotado de razón e intuición, conciencia y subconsciencia, instinto y espiritualidad y como ser histórico, avanza hacia la libertad en la creación de una nueva cultura y valores nuevos. Igual que todos los hombres, en su despliegue no es, sino que está siendo y dentro de sus retos están los de la integración y la liberación, por supuesto motivado por la búsqueda de la identidad. ${ }^{26}$

Al fin del camino se levantan los valores universales de la libertad y la solidaridad. Hoy, cuando la economía es global, transnacional y se orienta el mercantilismo corporativo y la lógica es la razón instrumental.
Así, frente a los desafíos de la propia realidad latinoamericana, nicaragüense y mundial, el filósofo Alejandro Serrano Caldera, produce todo un discurso filosófico que podemos decir es una filosofía de síntesis en una época de cambios en la visión del mundo y de la condición humana.

\section{Bibliografía}

1976 Introducción al pensamiento dialéctico. Fondo de Cultura Económica, México, D.F.

1979 Dialéctica y enajenación. EDUCA, San José, Costa Rica.

1984 Filosofía y crisis. Editorial Nueva Nicaragua, Managua.

1985 Entre la Nación y el Imperio. Editorial Vanguardia. Managua. Nicaragua.

1991 El Fin de la Historia: Reaparición de un mito. Editorial 13 de marzo, Universidad de La Habana, Cuba.

1993 La unidad en la diversidad. Hacia la Cultura del Consenso.

1994 El doble rostro de la postmodernidad. CSUCA, San José, Costa Rica.

1995 Los dilemas de la democracia. UAM, Managua.

2000 Estado de Derecho y Derechos Humanos. Instituto de Derechos Humanos.

Universidad Nacional Autónoma de Nicaragua. UNANLeón.

2001 Hacia un proyecto de nación. Una década de pensamiento político. Fondo Editorial CIRA, Managua, Nicaragua.

2013 Obras. Volumen IV. Escritos sobre el pensamiento, la política y la cultura nicaragüense II. Escritos jurídicos. HISPAMER_CNU, Managua.. 